\title{
Predictions for high-energy real and virtual photon-photon scattering from color dipole BFKL-Regge factorization
}

\author{
N.N.Nikolaev ${ }^{1,2}$, J.Speth ${ }^{1}$ and V. R.Zoller ${ }^{3}$ \\ 1 Institut für Kernphysik, Forschungszentrum Jülich, \\ D-52425 Jülich, Germany \\ E-mail:kph154@ikp301.ikp.kfa-juelich.de \\ 2 L.D.Landau Institute for Theoretical Physics, Chernogolovka, Moscow Region 142 432, \\ Russia \\ ${ }^{3}$ Institute for Theoretical and Experimental Physics, \\ Moscow 117218, Russia \\ E-mail:zoller@heron.itep.ru
}

\begin{abstract}
High-energy virtual photon-virtual photon scattering can be viewed as interaction of small size color dipoles from the beam and target photons, which makes $\gamma^{*} \gamma^{*}, \gamma^{*} \gamma$ scattering at high energies (LEP, LEP200 \& NLC) an indispensable probe of short distance properties of the QCD pomeron exchange. Based on the color dipole representation, we investigate consequences for the $\gamma^{*} \gamma^{*}, \gamma^{*} \gamma$ scattering of the incorporation of asymptotic freedom into the BFKL equation which makes the QCD pomeron a series of isolated poles in the angular momentum plane. The emerging color dipole BFKL-Regge factorization allows us to relate in a model-independent way the contributions of each BFKL pole to $\gamma^{*} \gamma^{*}, \gamma^{*} \gamma$ scattering and DIS off protons. Numerical predictions based on our early works on color dipole BFKL phenomenology of DIS on protons are in a good agreement with the experimental data on the photon structure function $F_{2 \gamma}$ and most recent data on the $\gamma^{*} \gamma^{*} \operatorname{cross}$ section $\sigma^{\gamma^{*} \gamma^{*}}(Y)$ from OPAL and L3 experiments at LEP200. We discuss the role of non-perturbative dynamics and predict pronounced effect of the Regge-factorization breaking due to large unfactorizable non-perturbative corrections to the perturbative vacuum exchange. We comment on the salient features of the BFKL-Regge expansion for $\gamma^{*} \gamma^{*}, \gamma^{*} \gamma$ scattering including the issue of decoupling of subleading BFKL poles and the soft plus rightmost hard BFKL pole dominance .
\end{abstract}

\section{Introduction}

In this note we study scattering of virtual and real photons

$$
\gamma^{*}(q)+\gamma^{*}(p) \rightarrow X
$$


in the high-energy regime of large Regge parameter $1 / x$ which depends on virtualities of photons as

$$
\frac{1}{x}=\frac{W^{2}+Q^{2}+P^{2}}{Q^{2}+P^{2}+\mu^{2}} \gg 1 .
$$

and has correct parton model limit if either $Q^{2} \ll P^{2}$ or $P^{2} \ll Q^{2}$. In eq.(2) $W^{2}=(q+p)^{2}$ is the center-of-mass energy squared of colliding space-like photons $\gamma^{*}(q)$ and $\gamma^{*}(p)$ with virtualities $q^{2}=-Q^{2}$ and $p^{2}=-P^{2}$, respectively.

The recent strong theoretical [1, 2, 3, 4, 5, 6, 7] and experimental [1, 8, 9, 10, 11, 12] (see also a compilation in [13]) interest in high-energy $\gamma^{*} \gamma^{*}, \gamma^{*} \gamma, \gamma \gamma$ scattering stems from the fact that virtualities of photons give a handle on the size of color dipoles in the beam and target photons and, eventually, on short distance properties of the QCD pomeron exchange. For earlier development of the subject see the pioneering paper [14].

As noticed by Fadin, Kuraev and Lipatov in 1975 [15] and discussed in more detail by Lipatov in [16] the incorporation of asymptotic freedom into the BFKL equation [17] makes the QCD pomeron a series of isolated poles in the angular momentum plane. The contribution of each isolated pole to the high-energy scattering amplitude satisfies the familiar Regge factorization [18]. In [19] we reformulated the consequences of the Regge factorization in our color dipole (CD) approach to the BFKL pomeron. In this communication we address several closely related issues in photon-photon scattering in the Regge regime (21) which can be tested at LEP200 and Next Linear Collider (NLC).

First, following our early work [19, 20, 21] we discuss how the color dipole (CD) BFKLRegge factorization leads to the parameter-free predictions for total cross sections of $\gamma^{*} \gamma^{*}$, $\gamma^{*} \gamma, \gamma \gamma$ scattering. We find good agreement with the recent experimental data from the L3 and OPAL experiments at LEP [8, 9, 10, 11, 12].

Second, we discuss the interplay of soft and hard dynamics of the vacuum exchange and comment on the onset of the soft plus rightmost hard BFKL-pole dominance in $\gamma^{*} \gamma^{*}$ diffractive scattering. The nodal properties of eigenfunctions of the CD BFKL equation suggest an interesting possibility of decoupling of sub-leading BFKL singularities when the virtuality of one or both of photons is in the broad vicinity of $Q^{2} \sim 20 \mathrm{GeV}^{2}$. Hence, very efficient the leading hard plus soft approximation (LHSA) advocated by us previously in 21.

Third, we discuss the impact of running CD BFKL on contentious issue of testing the factorization properties of photon-photon scattering in the $Q^{2}, P^{2}$-plane which has earlier been discussed only in the approximation of $\alpha_{S}=$ const to the BFKL equation [2, 3, [4 (for the color dipole picture in the $\alpha_{S}=$ const approximation see [22]). Our finding is that the non-perturbative corrections break down the Regge factorization. The experimental observation of this phenomenon would contribute to better understanding of the non-perturbative dynamics of high-energy processes.

\section{Overview of color dipole BFKL-Regge factorization}

In the color dipole basis the beam-target scattering is viewed as transition of $\gamma^{*}$ into quarkantiquark pair and interaction of the beam $(A)$ and target $(B)$ color dipoles of the flavor $A, B=u, d, s, c$. As a fundamental quantity we use the forward dipole scattering amplitude

and/or the dipole-dipole cross section $\sigma\left(x, \mathbf{r}, \mathbf{r}^{\prime}\right)$. Once $\sigma\left(x, \mathbf{r}, \mathbf{r}^{\prime}\right)$ is known the total cross 
section of $A B$ scattering $\sigma^{A B}(x)$ is calculated as

$$
\sigma^{b t}(x)=\int d z d^{2} \mathbf{r} d z^{\prime} d^{2} \mathbf{r}^{\prime}\left|\Psi_{A}(z, \mathbf{r})\right|^{2}\left|\Psi_{B}\left(z^{\prime}, \mathbf{r}^{\prime}\right)\right|^{2} \sigma\left(x, \mathbf{r}, \mathbf{r}^{\prime}\right)
$$

where $\mathbf{r}$ and $\mathbf{r}^{\prime}$ are the two-dimensional vectors in the impact parameter plane. In the color dipole factorization formula (3) the dipole-dipole cross section $\sigma\left(x, \mathbf{r}, \mathbf{r}^{\prime}\right)$ is beam-target symmetric and universal for all beams and targets, the beam and target dependence is concentrated in probabilities $\left|\Psi_{A}(z, \mathbf{r})\right|^{2}$ and $\left|\Psi_{B}\left(z^{\prime}, \mathbf{r}^{\prime}\right)\right|^{2}$ to find a color dipole, $\mathbf{r}$ and $\mathbf{r}^{\prime}$ in the beam and target, respectively. Hereafter we focus on cross sections averaged over polarizations of the beam and target photons, in this case only the term $n=0$ of the Fourier series

$$
\sigma\left(x, \mathbf{r}, \mathbf{r}^{\prime}\right)=\sum_{n=0}^{\infty} \sigma_{n}\left(x, r, r^{\prime}\right) \exp (i n \varphi)
$$

where $\varphi$ is an azimuthal angle between $\mathbf{r}$ and $\mathbf{r}^{\prime}$, contributes in (3).

Fadin, Kuraev and Lipatov noticed in 1975 [15], see also Lipatov's extensive discussion [16] that the incorporation of asymptotic freedom into the BFKL equation makes the QCD pomeron a series of isolated poles in the angular momentum plane. The contribution of the each pole to scattering amplitudes satisfies the standard Regge-factorization [18], which in the CD basis implies the CD BFKL-Regge expansion for the vacuum exchange dipole-dipole cross section

$$
\sigma\left(x, r, r^{\prime}\right)=\sum_{m} C_{m} \sigma_{m}(r) \sigma_{m}\left(r^{\prime}\right)\left(\frac{x_{0}}{x}\right)^{\Delta_{m}} .
$$

Here the dipole cross section $\sigma_{m}(r)$ is an eigen-function of the CD BFKL equation [19, 20, 23, 24, 25,

$$
\frac{\partial \sigma_{m}(x, r)}{\partial \log (1 / x)}=\mathcal{K} \otimes \sigma_{m}(x, r)=\Delta_{m} \sigma_{m}(x, r),
$$

with eigen value (intercept) $\Delta_{m}$. Arguably, for transition of $\gamma^{*}$ into heavy flavors, $A=c, b, \ldots$, the hardness scale is set by $Q^{2}+4 m_{A}^{2}$, for light flavors $Q^{2}+m_{\rho}^{2}$ is a sensible choice which leads to the correct value of Regge parameter in the photoproduction regime, $Q^{2} \rightarrow 0$.

Hence, for the light-light transition we evaluate the Regge-parameter (2) with $\mu^{2}=m_{\rho}^{2}$, for the light-charm contribution we take $\mu^{2}=4 m_{c}^{2}$ and for the charm-charm contribution we take $\mu^{2}=8 m_{c}^{2}$

For the details on CD formulation of the BFKL equation, infrared regularization by finite propagation radius $R_{c}$ for perturbative gluons and freezing of strong coupling at large distances, the choice of the physically motivated boundary condition for the hard BFKL evolution and description of eigenfunctions we refer to our early works [19, 20, 25, the successful application of CD BFKL-Regge expansion to the proton and pion structure functions (SF) and evaluation of hard-pomeron contribution to the rise of hadronic and real photo-absorption cross sections is found in [19, 20, 21, 25]. We only recapitulate the salient features of the formalism essential for the present discussion.

There is a useful analogy between the intercept $\Delta=\alpha(0)-1$ and binding energy for the bound state problem for the Schrödinger equation. The eigenfunction $\sigma_{0}(r)$ for the rightmost hard BFKL pole (ground state) corresponding to the largest intercept $\Delta_{0} \equiv \Delta_{\text {IP }}$ is node free. The eigenfunctions $\sigma_{m}(r)$ for excited states with $m$ radial nodes have intercept $\Delta_{m}<\Delta_{\mathbb{P}}$. Our choice of $R_{c}=0.27 \mathrm{fm}$ yields for the rightmost hard BFKL pole the intercept $\Delta_{\mathbb{P}}=0.4$, for sub-leading hard poles $\Delta_{m} \approx \Delta_{0} /(m+1)$. The node of $\sigma_{1}(r)$ is located at 
$r=r_{1} \simeq 0.05-0.06 \mathrm{fm}$, for larger $m$ the rightmost nodes move to a somewhat larger $r$ and accumulate at $r \sim 0.1 \mathrm{fm}$, for the more detailed description of the nodal structure of $\sigma_{m}(r)$ see [19, 20]. Here we only emphasize that for solutions with $m \geq 3$ the third and higher nodes are located at a very small $r$ way beyond the resolution scale $1 / \sqrt{Q^{2}}$ of foreseeable deep inelastic scattering (DIS) experiments. Notice that the Regge cut in the complex angular momentum plane found in the much discussed approximation $\alpha_{S}=$ const resembles an infinite, and continuous, sequence of poles. In the counterpart of our CD BFKL-Regge expansion (5) for approximation $\alpha_{S}=$ const the intercept $\Delta_{m}$ would be a continuous parameter in contrast to a discrete spectrum for standard running $\alpha_{S}$.

Because the BFKL equation sums cross sections of production of multigluon final states, the perturbative two-gluon Born approximation is an arguably natural boundary condition. This leaves the starting point $x_{0}$ as the only free parameter which fixes completely the result of the hard BFKL evolution for dipole-dipole cross section. We follow the choice $x_{0}=0.03$ made in 25]. The very ambitious program of description of $F_{2 p}\left(x, Q^{2}\right)$ starting from this, perhaps excessively restrictive, perturbative two-gluon boundary condition has been launched by us in [19] and met with remarkable phenomenological success [20, 21].

Because in the attainable region of $r$ the sub-leading solutions $m \geq 3$ can not be resolved and they all have similar intercepts $\Delta_{m} \ll 1$, in practical evaluation of $\sigma^{A B}$ we can truncate expansion (5) at $m=3$ lumping in the term with $m=3$ contributions of all singularities with $m \geq 3$. Specifically, if we endow

$$
\sigma_{3}(r)=\sigma_{B o r n}(r)-\sum_{m=0}^{2} \sigma_{m}(r)
$$

with the effective intercept $\Delta_{3}=0.06$ the truncated expansion reproduces the numerical solution $\sigma(x, r)$ of our CD BFKL equation in the wide range of dipole sizes $10^{-3} \lesssim r \lesssim 10$ fm with accuracy $\simeq 10 \%$ even at moderately small $x$. Such a truncation can be justified a posteriori if such a contribution from $m \geq 3$ turns out to be a small correction, which will indeed be the case at small $x$.

Whereas scattering of small dipoles $r \lesssim R_{c}$ is dominated by the exchange of perturbative gluons, interaction of large dipoles with the proton target has been modeled in Ref. [25, 20, 21 by the non-perturbative soft pomeron with intercept $\alpha_{\text {soft }}(0)-1=\Delta_{\text {soft }}=0$. Then the extra term $\sigma_{\text {soft }}\left(r, r^{\prime}\right)$ must be added in the r.h.s. of expansion (5).

From the early phenomenology of DIS and diffractive vector meson production off the proton target we only know the parameterization of $\sigma_{\text {soft }}\left(r, r^{\prime}\right)$ when one of the dipoles is definitely large, of the order of the proton size. Evaluation of the soft contribution to $\gamma^{*} \gamma^{*}$ scattering when both dipoles are small inevitably introduces model dependence. Modelling of soft contribution by the exchange by two nonperturbative gluons suggests [26]

$$
\sigma_{\text {soft }}\left(r, r^{\prime}\right) \propto \frac{r^{2} r^{\prime 2}}{\left(r^{2}+r^{\prime 2}\right)}
$$

and the non-factorizable cross section of the form

$$
\sigma_{\text {soft }}^{\gamma^{*} \gamma^{*}}\left(Q^{2}, P^{2}\right) \propto \frac{1}{Q^{2}+P^{2}}
$$

Similar non-perturbative $\sigma_{\text {soft }}$ is found in the soft pomeron models [27]. The explicit parameterization is found in Appendix. 
Finally, at moderately small values of $x$ the above described $t$-channel gluon tower exchange must be complemented by the $t$-channel $q \bar{q}$ exchange often associated with DIS off vector mesons (hadronic component) and off the perturbative (point-like) $q \bar{q}$-component of the target photon wave function. We add corresponding corrections only to the real photon structure function $F_{2 \gamma}\left(x, Q^{2}\right)$ to estimate the interplay of vacuum and non-vacuum exchanges in the currently accessible kinematical region of not very small $x$. In all other cases of interst we concentrate on the pure vacuum exchange at $x \lesssim x_{0}$ where the non-vacuum corrections are negligible small.

In our evaluation of the box diagram contribution to $F_{2 \gamma}^{p l}\left(x, Q^{2}\right)$ which is 29

$$
F_{2 \gamma}^{p l}\left(x, Q^{2}\right)=\frac{3 \alpha_{e m}}{\pi} \sum_{q=u d s c} e_{q}^{4} x\left\{\left[x^{2}+(1-x)^{2}\right] \log \frac{Q^{2}(1-x)}{x Q_{q}^{2}}+8 x(1-x)-1\right\}
$$

we take the $\rho$-meson mass as the lower cut-off for the light-flavor-loop integral, $Q_{q}^{2}=m_{\rho}^{2}$ for $q=u, d, s$, and the charm quark mass for the $c$-loop, $Q_{c}^{2}=m_{c}^{2}$. In eq.(8) $e_{q}$ is a quark charge.

To describe the hadronic component of $F_{2 \gamma}$ we take the coherent mixture of $\rho^{0}$ and $\omega$ mesons [30]. Being supplemented with quite standard assumptions on the vector meson valence quark density this gives

$$
\left.F_{2 \gamma}^{h a d}(x)=\frac{\alpha_{e m}}{12}\left[4\left(g_{\rho}+g_{\omega}\right)^{2}+\left(g_{\rho}-g_{\omega}\right)^{2}\right)\right] \sqrt{x}(1-x)
$$

where the coupling constants $g_{V}^{2}=4 \pi / f_{V}^{2}$, in the Fock state expansion

$$
|\gamma\rangle^{h a d}=\frac{e}{f_{\rho}}|\rho\rangle+\frac{e}{f_{\omega}}|\omega\rangle+\ldots
$$

are as follows $g_{\rho}^{2}=0.5$ and $g_{\omega}^{2}=0.043$ [13. We neglect the $Q^{2}$-evolution which, at reasonable values of lower scale, is a small correction on the interval $1.9 \leq Q^{2} \leq 5 \mathrm{GeV}^{2}$ where the small- $x$ data on $F_{2 \gamma}$ were taken.

Then, combining (5) and (3) and adding in the soft and non-vacuum components, we obtain our principal result for virtual-virtual scattering $(m=0,1,2,3, A, B=u, d, s, c)$

$$
\begin{array}{r}
\sigma_{\text {vac }}^{\gamma^{*} \gamma^{*}}\left(x, Q^{2}, P^{2}\right)=\frac{\left(4 \pi^{2} \alpha_{e m}\right)^{2}}{Q^{2} P^{2}} \sum_{m} C_{m} \sum_{A, B} f_{m}^{A}\left(Q^{2}\right) f_{m}^{B}\left(P^{2}\right)\left(\frac{3 x_{0}}{2 x_{A B}}\right)^{\Delta_{m}} \\
+\sigma_{\text {soft }}^{\gamma^{*} \gamma^{*}}\left(x, Q^{2}, P^{2}\right) .
\end{array}
$$

To make explicite the scale dependence discussed in Introduction we provide $\mu$ and $x$ defined by the eq.(2) with two indices, $A$ and $B$, pointing out the flavor of both the beam and target dipoles: $\mu_{A B}^{2}=m_{\rho}^{2}$ for $A, B=u, d, s$ while $\mu_{A B}^{2}=4 m_{c}^{2}$ if either $A=c$ or $B=c$ and the second dipole is made of light quarks and $\mu_{A B}^{2}=8 m_{c}^{2}$ if $A=B=c$.

For the DIS off real (quasireal) photons, $P^{2} \simeq 0$, we have $(A=u, d, s, c)$

$$
F_{2 \gamma}\left(x, Q^{2}\right)=\sum_{m} A_{m}^{\gamma} \sum_{A} f_{m}^{A}\left(Q^{2}\right)\left(\frac{3}{2} \frac{x_{0}}{x_{A}}\right)^{\Delta_{m}}+F_{2 \gamma}^{\mathrm{soft}}\left(x, Q^{2}\right)+F_{2 \gamma}^{\mathrm{nvac}}\left(x, Q^{2}\right),
$$

where

$$
x_{A}=\frac{Q^{2}+\mu_{A}^{2}}{W^{2}+Q^{2}}
$$


and $\mu_{A}^{2}=m_{\rho}^{2}$ for $A=u, d, s$ while $\mu_{A}^{2}=4 m_{c}^{2}$ for $A=c$. The $c \bar{c}$ component of the target photon wave function is strongly suppressed at $P^{2} \simeq 0$ and for all the practical purposes can be neglected as well as $c \bar{c}$ content of the target proton. This observation simplifies the factorization relation (11) for the real photon structure function. In eq.(11) the non-vacuum component denoted by $F_{2 \gamma}^{\text {nvac }}$ is

$$
F_{2 \gamma}^{\mathrm{nvac}}\left(x, Q^{2}\right)=F_{2 \gamma}^{\mathrm{had}}\left(x, Q^{2}\right)+F_{2 \gamma}^{\mathrm{pl}}\left(x, Q^{2}\right)
$$

and the cross sections

$$
\sigma_{m}^{\gamma^{*}}\left(Q^{2}\right)=\left\langle\gamma_{T}^{*}\left|\sigma_{m}(r)\right| \gamma_{T}^{*}\right\rangle+\left\langle\gamma_{L}^{*}\left|\sigma_{m}(r)\right| \gamma_{L}^{*}\right\rangle
$$

are calculated with the well known color dipole distributions in the transverse $(\mathrm{T})$ and longitudinal (L) photon of virtuality $Q^{2}$ derived in [28], and the eigen SFs are defined as usual:

$$
f_{m}\left(Q^{2}\right)=\frac{Q^{2}}{4 \pi^{2} \alpha_{\mathrm{em}}} \sigma_{m}^{\gamma^{*}}\left(Q^{2}\right) .
$$

The factor $\frac{3}{2}$ in the Regge parameter derives from the point that in a scattering of color dipole on the photon the effective dipole-dipole collision energy is $\frac{3}{2}$ of that in the reference scattering of color dipole on the three-quark nucleon at the same total c.m.s. energy $W$. The analytical formulas for the eigen-SFs $f_{m}\left(Q^{2}\right)$ and $f_{m}^{c}\left(Q^{2}\right)$ are found in the Appendix. Here as well as in all our previous calculations we put $m_{c}=1.5 \mathrm{GeV}$. We do not need any new parameters compared to those used in the description of DIS and real photoabsorption on protons [19, 20, 21] (for an alternative approach see [31, 32]), the results for the expansion parameters $A_{m}^{\gamma}=C_{m} \sigma_{m}^{\gamma}, C_{m}=1 / \sigma_{m}^{p}$ and $\sigma_{m}^{\gamma} \equiv \sigma_{m}^{\gamma^{*}}(0)$ are summarized in the Table 1 .

We recall that because of the diffusion in color dipole space, exchange by perturbative gluons contributes also to interaction of large dipoles $r>R_{c}$ [24]. However at moderately large Regge parameter this hard interaction driven effect is still small. For this reason in what follows we refer to terms $m=0,1,2,3$ as hard contribution as opposed to the genuine soft interaction.

Table 1. CD BFKL-Regge expansion parameters.

\begin{tabular}{|c|c|c|c|c|c|c|}
\hline$m$ & $\Delta_{m}$ & $\sigma_{m}^{p}, \mathrm{mb}$ & $C_{m}, \mathrm{mb}^{-1}$ & $A_{m}^{\gamma} / \alpha_{\mathrm{em}}$ & $\sigma_{m}^{\gamma}, \mu \mathrm{b}$ & $\sigma_{m}^{\gamma \gamma}, \mathrm{nb}$ \\
\hline 0 & 0.402 & 1.243 & 0.804 & 0.746 & 6.767 & 36.84 \\
\hline 1 & 0.220 & 0.462 & 2.166 & 0.559 & 1.885 & 7.69 \\
\hline 2 & 0.148 & 0.374 & 2.674 & 0.484 & 1.320 & 4.65 \\
\hline 3 & 0.06 & 3.028 & 0.330 & 0.428 & 9.456 & 29.53 \\
\hline soft & 0. & 31.19 & 0.0321 & 0.351 & 79.81 & 204.2 \\
\hline
\end{tabular}

\section{Isolating the soft plus rightmost hard BFKL pole in highly virtual-virtual $\gamma^{*} \gamma^{*}$ scattering}

We start with the theoretically cleanest case of the highly virtual photons, $P^{2}, Q^{2} \gg 1 \mathrm{GeV}^{2}$ and focus on the vacuum exchange component of the total cross section. The CD BFKL approach with asymptotic freedom predicts uniquely that subleading eigen SFs have a node at $Q^{2} \sim 20 \mathrm{GeV}^{2}$ in which region of $Q^{2}$ the rightmost hard pole contribution will dominate. 
This suppression of the subleading hard background is shown in Fig. 1, in which we plot the ratio $(m=0,1,2,3$, soft $)$

$$
r_{m}\left(Q^{2}\right)=\frac{\sigma_{m}^{\gamma^{*} \gamma^{*}}\left(\frac{3}{2} x_{0}, Q^{2}, Q^{2}\right)}{\sigma_{\mathrm{vac}}^{\gamma^{*} \gamma^{*}}\left(\frac{3}{2} x_{0}, Q^{2}, Q^{2}\right)},
$$

which defines the relative size of different contributions to $\sigma_{\mathrm{vac}}^{\gamma^{*} \gamma^{*}}$ at $x=\frac{3}{2} x_{0}$. At this value of $x$ the contribution of subleading hard BFKL poles remains marginal in a broad range of $Q^{2}$, although the contribution from the single-node component $m=1$ becomes substantial at $Q^{2} \gtrsim 10^{3} \mathrm{GeV}^{2}$.

The soft-pomeron exchange contributes substantially over all $Q^{2}$ and dominates at $Q^{2} \lesssim$ $1 \mathrm{GeV}^{2}$. However, at very large $W \sim 100 \mathrm{GeV}$ of the practical interest at LEP and LHC, such small values of $Q^{2}$ correspond to very small $x$, where the soft and subleading hard contributions are Regge suppressed by the factor $\left(x / x_{0}\right)^{\Delta_{\mathbf{I}}}$ and $\left(x / x_{0}\right)^{0.5 \Delta_{\mathbf{I P}}}$, respectively. The latter is clearly seen from Fig. 2 where the effective pomeron intercept

$$
\Delta_{e f f}=-\frac{\partial \log \sigma_{\mathrm{vac}}^{\gamma^{*} \gamma^{*}}}{\partial \log x}
$$

is presented for the diagonal case $Q^{2}=P^{2}$ at three different values of $W$.

According to the results shown in Fig. 1 the dominance of the soft plus rightmost hard BFKL pomeron exchange in virtual-virtual $\gamma^{*} \gamma^{*}$ scattering holds in a very broad range of $Q^{2}, P^{2} \lesssim 500 \mathrm{GeV}^{2}$ which nearly exhausts the interesting kinematical region at LEP200 and NLC. The quality of the leading hard pole plus soft approximation (LHSA) can be judged also from Fig. 3 for the diagonal case of $Q^{2}=P^{2}$, in which we show separately the soft component of the cross section (the dashed curve). The point that the contribution from subleading hard BFKL exchange is marginal is clear from the finding that approximation of soft-pomeron plus the rightmost hard BFKL exchange (LHSA) shown by long-dashed curve nearly exhausts the result from the complete CD BFKL-Regge expansion for vacuum exchange.

Recently the L3 collaboration [10 reported the first experimental evaluation of the vacuum exchange in equal virtuality $\gamma^{*} \gamma^{*}$ scattering. Their procedure of subtraction of the non-vacuum reggeon and/or the Quark Parton Model contribution is described in [10, arguably the subtraction uncertainties are marginal within the present error bars. In Fig. 4 we compare our predictions to the L3 data. The experimental data and theoretical curves are shown vs. the variable $Y=\log \left(W^{2} / \sqrt{Q^{2} P^{2}}\right)$. The virtuality of two photons varies in the range of $1.2 \mathrm{GeV}^{2}<Q^{2}, P^{2}<9 \mathrm{GeV}^{2}\left(\left\langle Q^{2}, P^{2}\right\rangle=3.5 \mathrm{GeV}^{2}\right)$ at $\sqrt{s} \simeq 91 \mathrm{GeV}$ and $2.5 \mathrm{GeV}^{2}<Q^{2}, P^{2}<35 \mathrm{GeV}^{2}$ at $\sqrt{s} \simeq 183 \mathrm{GeV}\left(\left\langle Q^{2}, P^{2}\right\rangle=14 \mathrm{GeV}^{2}\right)$. We applied to the theoretical cross sections the same averaging procedure as described in [10]. The solid curve is a result of the complete BFKL-Regge expansion for the vacuum exchange, the long-dashed curve is a sum of the rightmost hard BFKL exchange and soft-pomeron exchange. Shown by the dashed line is the soft pomeron contribution. The agreement of our estimates with the experiment is good, the contribution of subleading hard BFKL exchange is negligible within the experimental error bars.

In Fig. 5 we compare our predictions for the vacuum exchange contribution to $\sigma^{\gamma^{*} \gamma^{*}}(Y)$ with recent OPAL Collaboration measurements [12]. In the applicability region of our approach corresponding to $Y \gtrsim Y_{0}=\log \left(2 / 3 x_{0}\right) \simeq 3$ the agreement with data is good. The discrepancy at smaller $Y$ may indicate significant non-vacuum contributions vanishing at large $Y$. 
The early calculations [4, 2, 3] of the perturbative vacuum component of $\sigma^{\gamma^{*} \gamma^{*}}$ used the approximation $\alpha_{S}=$ const which predicts the $P^{2}, Q^{2}$-dependence different from our result for CD BFKL approach with running $\alpha_{S}$. Detailed comparison with numerical results by Brodsky, Hautmann and Soper (BHS) [4] is reported by the L3 Collaboration [10, which founds that BHS formulas overpredict $\sigma_{\mathrm{vac}}^{\gamma^{*} \gamma^{*}}$ substantially. In [3] the same perturbative fixed- $\alpha_{S}$ BFKL model with massive $c$-quark has been considered. At $\left\langle Q^{2}\right\rangle=14 \mathrm{GeV}^{2}$ and moderately small $x, x \gtrsim 3.10^{-2}$, the model is in agreement with the L3 data but at smaller $x$, already at $x \sim 7.10^{-3}$, it substanially overpredicts $\sigma_{\text {vac }}^{\gamma^{*} \gamma^{*}}$. At $\left\langle Q^{2}\right\rangle=3.5 \mathrm{GeV}^{2}$ the results [3] are substantially above the L3 data over all $x$.

\section{Virtual-real $\gamma^{*} \gamma$ scattering: the rightmost hard BFKL pole in the photon SF}

The discussion of the photon SF follows closely that of the proton and pion SF's in [19, 20, 21]. Our normalization of eigen-functions is such that the vacuum (sea) contribution to the proton $\mathrm{SF}(m=$ soft, $0,1, . ., 3)$

$$
F_{2 p}\left(x, Q^{2}\right)=\sum_{m} f_{m}\left(Q^{2}\right)\left(\frac{x_{0}}{x}\right)^{\Delta_{m}}
$$

has the CD BFKL-Regge expansion coefficients $A_{m}^{p}=1$. There is a fundamental point that the distribution of small-size color dipoles in the photon is enhanced compared to that in the proton [21] which enhances the importance of the rightmost hard BFKL exchange. Indeed, closer inspection of expansion coefficients $A_{m}^{\gamma}$ shown in table 1 reveals that subleading hard BFKL exchanges are suppressed with respect to the leading one by the factor $\simeq 1.5$, whereas the soft-pomeron exchange contribution is suppressed by the factor $\simeq 2$.

Our predictions for the photon SF are parameter-free and are presented in Fig. 6. At moderately small $x \sim 0.1$ there is a substantial non-vacuum reggeon exchange contribution from DIS off hadronic $(q \bar{q})$ component of the target photon wave function which can be regarded as well constrained by the large $x$ data. We use here the parameterizations presented above (eqs. (9,8, 12)). The solid curve shows the result from the complete BFKLRegge expansion the soft-pomeron (the dashed curve) and quasi-valence (the dot-dashed curve) components included, the dotted curve shows the rightmost hard BFKL (LH) plus soft-pomeron (S) plus non-vacuum (NV) approximation (LHSNVA). A comparison of the solid and dotted curves shows clearly that subleading hard BFKL exchanges are numerically small in the experimentally interesting region of $Q^{2}$, the rightmost hard BFKL pole exhausts the hard vacuum contribution for $2 \lesssim Q^{2} \lesssim 100 \mathrm{GeV}^{2}$. The nodal properties of subleading hard BFKL SFs are clearly seen: LHSNVA underestimates $F_{2 \gamma}$ slightly at $Q^{2} \lesssim 10 \mathrm{GeV}^{2}$ and overestimates $F_{2 \gamma}$ at $Q^{2} \gtrsim 50 \mathrm{GeV}^{2}$. For still another illustration of the same nodal property of subleading hard components see Fig. 7 in which we show the vacuum component of virtual-real total cross section $\sigma_{t o t}^{\gamma^{*} \gamma}$ as a function of $Q^{2}$ at fixed $W$. As seen from Fig. 1, the soft contribution rises towards small $Q^{2}$, but this rise is compensated to a large extent by the small- $x$ enhancement of the rightmost hard BFKL contribution by the large Regge factor $\left(\frac{x_{0}}{x}\right)^{\Delta_{\mathbb{P}}}$. For this region the soft background (the dashed curve) remains marginal over the whole range of $Q^{2}$. Because of the node effect, the $m=1$ subleading component changes the sign and becomes quite substantial at very large $Q^{2}$ and moderately small $x$. 
Recently the L3 and OPAL collaborations reported the first experimental data on the photon SF at sufficiently small-x 8, 11]. These data are shown in Fig. 6 and are in good agreement with the predictions from the CD BFKL-Regge expansion. A comparison with the long-dashed curve which is the sum of the rightmost hard BFKL and soft exchanges shows that the experimental data are in the region of $x$ and $Q^{2}$ still affected by non-vacuum reggeon (quasi-valence) exchange (dot-dashed line), going to smaller $x$ and larger $Q^{2}$ would improve the sensitivity to pure vacuum exchange greatly.

In order to give a crude idea on finite-energy effects at large $x$ and not so large values of the Regge parameter we stretch the theoretical curves a bit to $x \gtrsim x_{0}$ multiplying the BFKL-Regge expansion result by the purely phenomenological factor $(1-x)$ motivated by the familiar behavior of the gluon SF of the photon $\sim(1-x)^{n}$ with the exponent $n \sim 1$.

\section{$5 \quad$ The real-real $\gamma \gamma$ scattering}

We recall that because of the well known BFKL diffusion in color dipole space, exchange by perturbative gluons contributes also to interaction of large dipoles $r>R_{c}$ [24]. As discussed in [21] this gives rise to a substantial rising component of hadronic and real photoabsorption cross sections and a scenario in which the observed rise of hadronic and real photon cross sections is entirely due to this intrusion of hard scattering. This is a motivation behind our choice of intercept $\Delta_{\text {soft }}=0$ for soft pomeron exchange. Furthermore, in order to make this picture quantitative one needs to invoke strong absorption/unitarization to tame too a rapid growth of large dipole component of hard BFKL the dipole cross section. The case of real-real $\gamma \gamma$ scattering is not an exception and the above discussed enhancement of small dipole configurations in photons compared to hadrons predicts uniquely that the hard BFKL exchange component of real-real $\gamma \gamma$ scattering will be enhanced compared to proton-proton and/or pion-proton scattering. This is clearly seen from table 1 in which we show the coefficients

$$
\sigma_{m}^{\gamma \gamma}=\sigma_{m}^{\gamma} \sigma_{m}^{\gamma} C_{m}
$$

of the expansion for the vacuum exchange component of the total $\gamma \gamma$ cross section $(m=$ $0,1,2,3$, soft)

$$
\sigma_{\text {vac }}^{\gamma \gamma}=\sum_{m} \sigma_{m}^{\gamma \gamma}\left(\frac{W^{2} x_{0}}{m_{\rho}^{2}}\right)^{\Delta_{m}} .
$$

One has to look at the soft-hard hierarchy of $\sigma_{m}^{\gamma \gamma}$ and $\sigma_{m}^{\gamma}, \sigma_{m}^{p}$ in the counterparts of (18) for $\gamma p$ and $p p$ scattering. This enhancement of hard BFKL exchange is confirmed by simplified vacuum pole plus non-vacuum reggeon exchange fits to real-real $\gamma \gamma$ total cross section: the found intercept of the effective vacuum pole $\epsilon^{\gamma \gamma} \approx 0.21$ is much larger than $\epsilon \approx 0.095$ from similar fits to the hadronic cross section data. In Fig. 8 we compare our predictions from the CD BFKL-Regge factorization for the single-vacuum exchange contribution to real-real $\gamma \gamma$ scattering with the recent experimental data from the OPAL collaboration [9] and [13]. The theoretical curves are in the right ballpark, but the truly quantitative discussion of total cross sections of soft processes requires better understanding of absorption/unitarization effects. 


\section{Regge factorization in $\gamma^{*} \gamma^{*}$ and $\gamma \gamma$ scattering}

If the vacuum exchange were an isolated Regge pole, the well known Regge factorization would hold for asymptotic cross sections [18]

$$
\sigma_{\text {tot }}^{b b} \sigma_{\text {tot }}^{a a}=\sigma_{\text {tot }}^{a b} \sigma_{\text {tot }}^{a b}
$$

In the CD BFKL approach such a Regge factorization holds for each term in the BFKLRegge expansion for vacuum exchange, but evidently the sum of factorized terms does not satisfy the factorization (19). One can hope for an approximate factorization only provided one single term to dominate in the BFKL-Regge expansion. Though corrections to the exact factorization still exist even for the single pole exchange because of the light $q \bar{q}$ and charm $c \bar{c}$ mass scale difference discussed above.

One such case is real-real $\gamma \gamma$ scattering dominated by soft-pomeron exchange (the factorization of the soft on-shell amplitudes though never proved gained strong support from the high-energy Regge-phenomenology). For this reason the CD BFKL-Regge expansion which reproduces well the vacuum exchange components of the $p p$ and $\gamma p$ scattering can not fail for the vacuum component in real-real $\gamma \gamma$ scattering. The rise of the contribution of hard-BFKL exchange breaks the Regge factorization relation

$$
R_{\gamma \gamma}=\frac{\sigma_{\mathrm{vac}}^{\gamma \gamma} \sigma^{p p}}{\sigma_{\mathrm{vac}}^{\gamma p} \sigma_{\mathrm{vac}}^{\gamma p}}=1
$$

which would restore at extremely high energies such that the rightmost hard BFKL exchange dominates. This property is illustrated in Fig. 9 where we show our evaluation of $R$ for singlevacuum component of total cross sections entering (19). At moderately high energies naive factorization breaks but the expected breaking is still weak, $\lesssim 20 \%$. This curve must not be taken at face value for $W \gtrsim 0.1-1 \mathrm{TeV}$ because of likely strong absorption effects, but the trend of $R$ being larger than unity and rising with energy should withstand unitarity effects. The second case is highly virtual-virtual $\gamma^{*} \gamma^{*}$ scattering. As we emphasized in section 3 , here the CD BFKL approach predicts uniquely that because of the nodal property of subleading eigen SFs the the superposition of soft and rightmost hard BFKL poles dominate the vacuum exchange in a broad range of $Q^{2}, P^{2} \lesssim 10^{3} \mathrm{GeV}^{2}$.

The above discussion suggests clearly that different cross sections must be taken at the same value of $x^{-1}=W^{2} /\left(Q^{2}+P^{2}\right)$, in which case the vacuum components of $\gamma^{*} \gamma^{*}$ scattering at $Q^{2}, P^{2} \gg 4 m_{c}^{2}$ and $x \ll x_{0}$ would satisfy

$$
R_{\gamma^{*} \gamma^{*}}(x)=\frac{\left[\sigma^{\gamma^{*} \gamma^{*}}\left(x, Q^{2}, P^{2}\right)\right]^{2}}{\sigma^{\gamma^{*} \gamma^{*}}\left(x, Q^{2}, Q^{2}\right) \sigma^{\gamma^{*} \gamma^{*}}\left(x, P^{2}, P^{2}\right)}=1 .
$$

In accordance to the results shown in Fig. 1, the soft exchanges break the factorization relation (21). The breaking is quite substantial at moderate $x=0.01$ (dotted line in Fig. 10), and breaking effects disappear rapidly, $\sim x^{\Delta_{0}}$, as $x \rightarrow 0$. If the vacuum singularity were the Regge cut as is the case in approximation $\alpha_{S}=$ const, then restoration of factorization is much slower, cf. our Fig. 10 and Fig. 9 in $\llbracket$.

For an obvious reason that the soft-pomeron exchange is so predominant in real photon scattering, whereas the soft plus rightmost hard BFKL exchange is outstanding in virtualvirtual and real-virtual photon-photon scattering, it is ill advised to look at factorization ratio $R_{\gamma^{*} \gamma^{*}}(W)$ when one of the photons is quasireal, $P^{2} \sim 0$. In this limit one would find 
strong departures of $R_{\gamma^{*} \gamma^{*}}(W)$ from unity. For precisely the same reason of predominance of soft-pomeron exchange in $p p$ scattering vs. nearly dominant rightmost hard BFKL pole exchange in DIS at small $x$ and $5-10 \lesssim Q^{2} \lesssim 100 \mathrm{GeV}^{2}$, see [21], the naive factorization estimate

$$
\sigma^{\gamma^{*} \gamma^{*}}\left(W, Q^{2}, P^{2}\right) \approx \frac{\sigma^{\gamma^{*} p}\left(W, Q^{2}\right) \sigma^{\gamma^{*} p}\left(W, P^{2}\right)}{\sigma^{p p}(W)}
$$

would not make much sense.

\section{Conclusions}

We explored the consequences for small- $x$ photon $\operatorname{SFs} F_{2 \gamma}\left(x, Q^{2}\right)$ and high-energy twophoton cross sections $\sigma^{\gamma^{*} \gamma^{*}}$ and $\sigma^{\gamma \gamma}$ from the color dipole BFKL-Regge factorization. Because of the nodal properties of eigen SFs of subleading hard BFKL exchanges the CD BFKL approach predicts uniquely that the vacuum exchange is strongly dominated by the combination of soft plus rightmost hard BFKL pole exchanges in a very broad range of photon virtualities $Q^{2}, P^{2}$ which includes much of the kinematical domain attainable at LEP200 and NLC. Starting with very restrictive perturbative two-gluon exchange as a boundary condition for BFKL evolution in the color dipole basis and having fixed the staring point of BFKL evolution in the early resulting CD BFKL-Regge phenomenology of the proton $\mathrm{SF}$, we presented parameter-free predictions for the vacuum exchange contribution to the photon structure function which agree well with OPAL and L3 determinations. A good agreement is found between our predictions for the energy and photon virtuality dependence of the photon-photon cross section $\sigma^{\gamma^{*} \gamma^{*}}\left(W, Q^{2}, P^{2}\right)$ and the recent data taken by the L3 Collaboration. We commented on the utility of Regge factorization tests of the CD BFKL-Regge expansion.

Acknowledgments: This work was partly supported by the grants INTAS-96-597 and INTAS-97-30494 and DFG 436RUS17/11/99.

\section{Appendix}

\subsection{BFKL all flavor eigen-SF}

In the early discussion of DIS off protons the results of numerical solutions of the CD BFKL equation for the all flavor $(u+d+s+c)$ eigen-SF $f_{m}\left(Q^{2}\right)$ were parameterized as

$$
\begin{gathered}
f_{0}\left(Q^{2}\right)=a_{0} \frac{R_{0}^{2} Q^{2}}{1+R_{0}^{2} Q^{2}}\left[1+c_{0} \log \left(1+r_{0}^{2} Q^{2}\right)\right]^{\gamma_{0}} \\
f_{m}\left(Q^{2}\right)=a_{m} f_{0}\left(Q^{2}\right) \frac{1+R_{0}^{2} Q^{2}}{1+R_{m}^{2} Q^{2}} \prod_{i=1}^{m}\left(1-\frac{z}{z_{m}^{(i)}}\right), m \geq 1,
\end{gathered}
$$

where $\gamma_{0}=\frac{4}{3 \Delta_{0}}$ and

$$
z=\left[1+c_{m} \log \left(1+r_{m}^{2} Q^{2}\right)\right]^{\gamma_{m}}-1, \gamma_{m}=\gamma_{0} \delta_{m}
$$


The parameters tuned to reproduce the numerical results for $f_{m}\left(Q^{2}\right)$ at $Q^{2} \lesssim 10^{5} \mathrm{GeV}^{2}$ are listed in the Table 2.

Table 2. CD BFKL-Regge the all flavor SF parameters.

\begin{tabular}{|l|l|l|l|l|l|l|l|l|}
\hline$m$ & $a_{m}$ & $c_{m}$ & $r_{m}^{2}, \mathrm{GeV}^{-2}$ & $R_{m}^{2}, \mathrm{GeV}^{-2}$ & $z_{m}^{(1)}$ & $z_{m}^{(2)}$ & $z_{m}^{(3)}$ & $\delta_{m}$ \\
\hline 0 & 0.0232 & 0.3261 & 1.1204 & 2.6018 & & & & 1. \\
\hline 1 & 0.2788 & 0.1113 & 0.8755 & 3.4648 & 2.4773 & & & 1.0915 \\
\hline 2 & 0.1953 & 0.0833 & 1.5682 & 3.4824 & 1.7706 & 12.991 & & 1.2450 \\
\hline 3 & 1.4000 & 0.04119 & 3.9567 & 2.7706 & 0.23585 & 0.72853 & 1.13044 & 0.5007 \\
\hline soft & 0.1077 & 0.0673 & 7.0332 & 6.6447 & & & & \\
\hline
\end{tabular}

The soft component of the proton structure function as derived from $\sigma_{\text {soft }}(r)$ taken from 333 is parameterized as follows

$$
f_{\text {soft }}\left(Q^{2}\right)=\frac{a_{\text {soft }} R_{\text {soft }}^{2} Q^{2}}{1+R_{\text {soft }}^{2} Q^{2}}\left[1+c_{\text {soft }} \log \left(1+r_{\text {soft }}^{2} Q^{2}\right)\right],
$$

with parameters cited in the Table 2 .

The cross section $\sigma_{\text {soft }}^{\gamma^{*} \gamma^{*}}\left(Q^{2}, P^{2}\right)$ obtained by the continuation of the above

$$
\sigma_{\text {soft }}^{\gamma^{*} p}=\frac{4 \pi^{2} \alpha_{e m}}{Q^{2}} f_{\text {soft }}\left(Q^{2}\right)
$$

into $Q^{2}, P^{2}$-plane reads

$$
\sigma_{\text {soft }}^{\gamma^{*} \gamma^{*}}\left(Q^{2}, P^{2}\right)=\frac{\sigma_{\text {soft }}^{\gamma \gamma}}{1+R_{\text {soft }}^{2}\left(Q^{2}+P^{2}\right)}\left[1+c_{\text {soft }} \log \left(1+\frac{r_{\text {soft }}^{2} Q^{2}}{1+r_{\text {soft }}^{2} P^{2}}+\frac{r_{\text {soft }}^{2} P^{2}}{1+r_{\text {soft }}^{2} Q^{2}}\right)\right],
$$

with parameters cited in the Table 2 and the on-shell cross section

$$
\sigma_{\mathrm{soft}}^{\gamma \gamma}=\left[4 \pi^{2} \alpha_{e m} a_{\mathrm{soft}} R_{\mathrm{soft}}^{2}\right]^{2} \frac{1}{\sigma_{\mathrm{soft}}^{p p}}
$$

\subsection{BFKL charm eigen-SF}

In practical evaluations one needs the charm eigen-SF, $f_{m}^{c}\left(Q^{2}\right)$. For the rightmost hard BFKL pole it is of the form

$$
f_{0}^{c}\left(Q^{2}\right)=a_{0} \frac{R_{0}^{2} Q^{2}}{1+R_{0}^{2} Q^{2}}\left[1+c_{0} \log \left(1+r_{0}^{2} Q^{2}\right)\right]^{\gamma_{0}}
$$

where $\gamma_{0}=4 /\left(3 \Delta_{0}\right)$, while for the sub-leading hard BFKL poles

$$
f_{m}^{c}\left(Q^{2}\right)=a_{m} f_{0}\left(Q^{2}\right) \frac{1+K_{m}^{2} Q^{2}}{1+R_{m}^{2} Q^{2}} \prod_{i=1}^{m_{\max }}\left(1-\frac{z}{z_{m}^{(i)}}\right), m \geq 1
$$

where $m_{\max }=\min \{m, 2\}$ and

$$
z=\left[1+c_{m} \log \left(1+r_{m}^{2} Q^{2}\right)\right]^{\gamma_{m}}-1, \gamma_{m}=\gamma_{0} \delta_{m}
$$


The parameters tuned to reproduce the numerical results for $f_{m}^{c}\left(Q^{2}\right)$ at $Q^{2} \lesssim 10^{5} \mathrm{GeV}^{2}$ are listed in the Table 3.

The soft component of the charm SF is parameterized as

$$
f_{\text {soft }}^{c}\left(Q^{2}\right)=\frac{a_{\text {soft }} R_{\text {soft }}^{2} Q^{2}}{1+R_{\text {soft }}^{2} Q^{2}}\left[1+c_{\text {soft }} \log \left(1+r_{\text {soft }}^{2} Q^{2}\right)\right],
$$

with parameters cited in the Table 3.

Table 3. CD BFKL-Regge charm structure functions parameters.

\begin{tabular}{|c|c|c|c|c|c|c|c|c|}
\hline$m$ & $a_{m}$ & $c_{m}$ & $\begin{array}{c}r_{m}^{2}, \\
\mathrm{GeV}^{-2}\end{array}$ & $\begin{array}{c}R_{m}^{2}, \\
\mathrm{GeV}^{-2}\end{array}$ & $\begin{array}{c}K_{m}^{2}, \\
\mathrm{GeV}^{-2}\end{array}$ & $z_{m}^{(1)}$ & $z_{m}^{(2)}$ & $\delta_{m}$ \\
\hline 0 & 0.02140 & 0.2619 & 0.3239 & 0.2846 & & & & 1. \\
\hline 1 & 0.0782 & 0.03517 & 0.0793 & 0.2958 & 0.2846 & 0.2499 & & 1.9249 \\
\hline 2 & 0.00438 & 0.03625 & 0.0884 & 0.2896 & 0.2846 & 0.0175 & 3.447 & 1.7985 \\
\hline 3 & -0.26313 & 2.1431 & $3.7424 \cdot 10^{-2}$ & $8.1639 \cdot 10^{-2}$ & 0.13087 & 158.52 & 559.50 & 0.62563 \\
\hline soft & 0.01105 & 0.3044 & 0.09145 & 0.1303 & & & & \\
\hline
\end{tabular}

\section{References}

[1] R.Nisius, Phys.Rep. 332 (2000) 165

[2] J. Bartels , A. De Roeck and H. Lotter, Phys.Lett.B389 (1996) 742

[3] J. Bartels, C. Ewerz and R. Staritzbichler, Effect of the charm quark mass on the BFKL $\gamma^{*} \gamma^{*}$ total cross-section at LEP. e-Print Archive: hep-ph/0004029

[4] S.J. Brodsky, F. Hautmann and D.E. Soper, Phys.Rev.D56 (1997) 6957

[5] M. Boonekamp, A. De Roek, Ch. Royon and S. Wallon, Nucl.Phys.B555 (1999) 540

[6] B. Badelek, M. Krawczyk, J. Kwiecinski, A. M. Stasto, Phys.Rev. D62 (2000) 074021

[7] J. Kwiecinski, L. Motyka, Eur.Phys.J. C18 (2000) 343

[8] L3 Coll., M.Acciarri et al., Phys. Lett. B436 (1998) 403

[9] OPAL Coll., G.Abbiendi et al., CERN-EP/99-076 submitted to Eur.Phys. J

[10] L3 Coll., M.Acciarri et al., Phys.Lett. B453 (1999) 333

[11] OPAL Coll., K.Ackerstaff et al., Phys. Lett. B412 (1997) 225

[12] OPAL Coll., G.Abbiendi et al., Measurement of the Hadronic Cross-Section for the Scattering of Two Virtual Photons at LEP, Report CERN-EP-Sptember 2001.

[13] Particle Data Group, Eur.Phys.J. C3 (1998) 1

[14] V.M. Budnev, I.F. Ginzburg, G.V.Meledin and V.G. Serbo, Phys.Rep. 15 (1975) 181 
[15] V.S.Fadin, E.A.Kuraev and L.N.Lipatov Phys. Lett. B60 (1975) 50; E.A.Kuraev, L.N.Lipatov and V.S.Fadin, Sov.Phys. JETP 44 (1976) 443; 45 (1977) 199

[16] L.N. Lipatov, Sov. Phys. JETP 63 (1986) 904

[17] L.N.Lipatov, Sov. J. Nucl. Phys 23 (1976) 338; E.A.Kuraev, L.N.Lipatov and V.S.Fadin, Sov. JETP 44 (1976) 443; ibid. 45 (1977) 199; Ya.Ya.Balitsky and L.N.Lipatov, Sov. J. Nucl. Phys 28 (1978) 822

[18] V.N.Gribov, B.L.Ioffe, I.Ya. Pomeranchuk and A.P. Rudik, Sov.Phys. JETP 16 (1963) 220

[19] N.N. Nikolaev, B.G. Zakharov, V.R. Zoller, JETP Letters 66 (1997) 138.

[20] N.N. Nikolaev and V.R. Zoller, JETP Letters 69 (1999) 103; 176.

[21] N.N. Nikolaev, J. Speth and V.R. Zoller, Phys. Lett. B473 (2000) 157

[22] A.H.Mueller, Nucl. Phys. B415 (1994) 373

[23] N.N. Nikolaev, B.G. Zakharov and V.R. Zoller, JETP Letters 59 (1994) 8

[24] N.N. Nikolaev, B.G. Zakharov and V.R. Zoller, JETP 105 (1994) 1498

[25] N.N.Nikolaev and B.G.Zakharov, Phys. Lett. B327 (1994) 149; B333 (1994) 250; B327 (1994) 157.

[26] E.M. Levin and M.G. Ryskin, Sov.J.Nucl.Phys 34 (1981) 619

[27] O.Nachtmann,Ann. Phys. 209 (1991) 436; H.Dosch and Yu.A.Simonov, Phys. Lett. B205 (1988) 339; Yu.A.Simonov, Nucl.Phys. B324 (1989) 67; H.Dosch, E.Ferreira and A.Krämer, Phys. Rev. D50 (1994) 1992. P.V.Landshoff and O.Nachtmann, Z. Phys. C35 (1987) 405; H.G.Dosch, T.Gousset, G.Kulzinger and H.J.Pirner Phys. Rev. D55 (1997) 2602. A. Donnachie and P.V. Landshoff, Phys.Lett. B437 (1998) 408; K. Golec-Biernat and M. Wüsthoff, Phys.Rev. D59 (1999) 014017 U.D'Alesio, A.Metze and H.J.Pirner, Eur.Phys.J. C9 (1999) 601; G.Kulzinger, H.G.Dosch and H.J.PirnerEur.Phys.J. C7 (1999) 73

[28] N.N. Nikolaev and B.G. Zakharov, Z. Phys. C49 (1991) 607.

[29] T.F.Walsh and P.M. Zerwas, Phys.Lett.44B (1973) 195; R.L.Kingsley,Nucl.Phys.B60 (1973) 45

[30] G.A. Schuler and T. Sjöstrand Phys.Lett.B376 (1996) 193

[31] A.J.Askew, K.Golec-Biernat, J.Kwiecinski, A.D.Martin and P.J.Sutton, Phys.Lett. B325 (1994) 212

[32] H.Navelet, R.Peshanski, C.Royon and S.Wallon, Phys.Lett. B385 (1996) 357

[33] J. Nemchik, N.N. Nikolaev, E. Predazzi, B.G. Zakharov and V.R. Zoller, JETP 86 (1998) 1054. 


\section{Figure Captions}

Fig.1 The normalized ratio of soft-to-rightmost-hard and subleading hard-to-rightmost hard expansion coefficients $(m=1,2,3$, soft $) r_{m}\left(Q^{2}\right)=\sigma_{m}^{\gamma^{*} \gamma^{*}} / \sigma_{\text {vac }}^{\gamma^{*} \gamma^{*}}$ of the BFKL-Regge expansion for $\gamma^{*} \gamma^{*}$ scattering at $x=x_{0}$.

Fig.2 Predictions from CD BFKL-Regge expansion for the effective intercept $\Delta_{\text {eff }}$, eq.(15), for the diagonal case $Q^{2}=P^{2}$ and $W=50,100,200 \mathrm{GeV}$.

Fig.3 Predictions from CD BFKL-Regge expansion for the vacuum exchange component of the virtual-virtial $\gamma^{*} \gamma^{*}$ cross section for the diagonal case of $Q^{2}=P^{2}$ and for cms collision energy $\mathrm{W}=50,100$ and $200 \mathrm{GeV}$ (solid curves). The Leading Hard BFKL exchnage plus Soft-pomeron exchange Approximation (LHSA) is shown by the long dashed curve. The soft pomeron component of the cross section is shown separately by the dashed curve.

Fig.4 Predictions from CD BFKL-Regge expansion for the vacuum exchange component of the virtual-virtial $\gamma^{*} \gamma^{*}$ cross section for the diagonal case of $\left\langle Q^{2}\right\rangle=\left\langle P^{2}\right\rangle$ are confronted to the experimental data by the L3 Collaboration [10]. The experimental data and theoretical curves are shown vs. the variable $Y=\log \left(W^{2} / \sqrt{Q^{2} P^{2}}\right)$. The solid curve shows the result from the complete BFKL-Regge expansion, the soft-pomeron (the dashed curve) component included. the long dashed curve shows the rightmost hard BFKL (LH) plus soft-pomeron (S) approximation (LHSA).

Fig.5 Predictions from CD BFKL-Regge expansion for the vacuum exchange component of the virtual-virtial $\gamma^{*} \gamma^{*}$ cross section for the diagonal case of $\left\langle Q^{2}\right\rangle=\left\langle P^{2}\right\rangle=17.9 \mathrm{GeV}^{2}$ are confronted to the experimental data by the OPAL Collaboration [12]. The experimental data and theoretical curves are shown vs. the variable $Y=\log \left(W^{2} / \sqrt{Q^{2} P^{2}}\right)$. The solid curve shows the result from the complete BFKL-Regge expansion, the softpomeron (the dashed curve) component included. The long dashed line corresponds to the rightmost hard BFKL (LH) plus soft-pomeron (S) approximation (LHSA).

Fig.6 Predictions from CD BFKL-Regge expansion for the photon SF. The solid curve shows the result from the complete BFKL-Regge expansion the soft-pomeron (the dashed curve) and valence (the dot-dashed curve) components included, the dotted curve shows the rightmost hard BFKL (LH) plus soft-pomeron (S) plus non-vacuum (NV) approximation (LHSNVA). The long dashed line corresponds to the LH plus S approximation (LHSA). Data points are from [8, 11]

Fig.7 Predictions from CD BFKL-Regge expansion for the vacuum exchange component of the the virtual-real $\gamma^{*} \gamma$ total cross section and for cms collision energy $\mathrm{W}=50,100$ and $200 \mathrm{GeV}$ (solid curves). The result from the rightmost Hard BFKL (LH) plus Soft-pomeron (S) Approximation (LHSA) is shown by the long dashed curve. The soft-pomeron exchange component of the cross section is shown separately by the dashed curve.

Fig.8 Our predictions from the CD BFKL-Regge factorization for the single-vacuum exchange contribution to real-real $\gamma \gamma$ scattering are compared with the recent experimental data from the OPAL collaboration [9] and [13]. 
Fig.9 Our evaluation of $R_{\gamma \gamma}$ for single-vacuum component of total cross sections.

Fig.10 The factorization cross section ratio $R_{\gamma^{*} \gamma^{*}}(x)$ at fixed $x$ and $Q P$ as a function of $Q / P$ for $x=10^{-2}$ (dotted line), $x=10^{-3}$ (long-dashed) and $x=10^{-4}$ (dashed). 


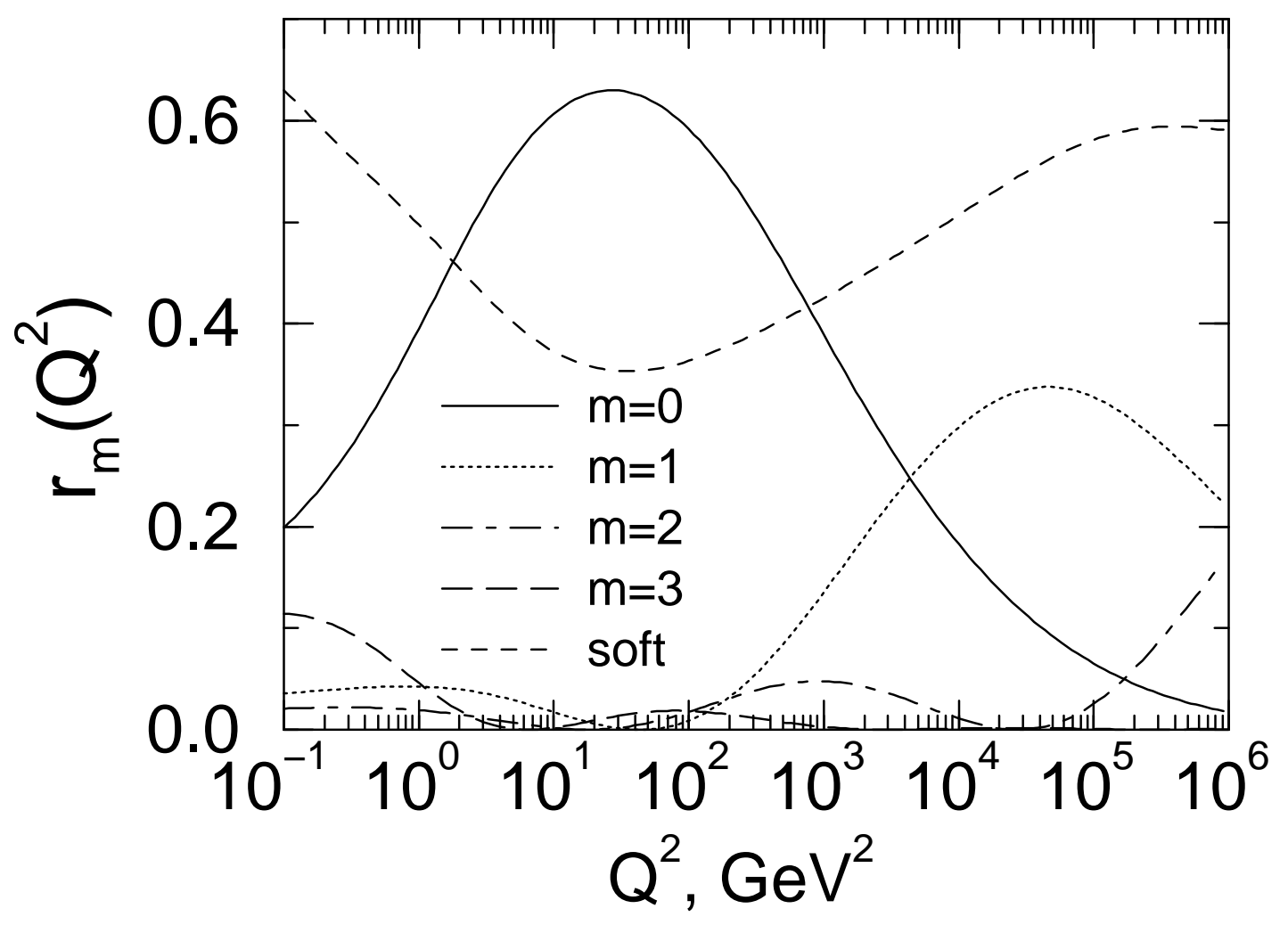




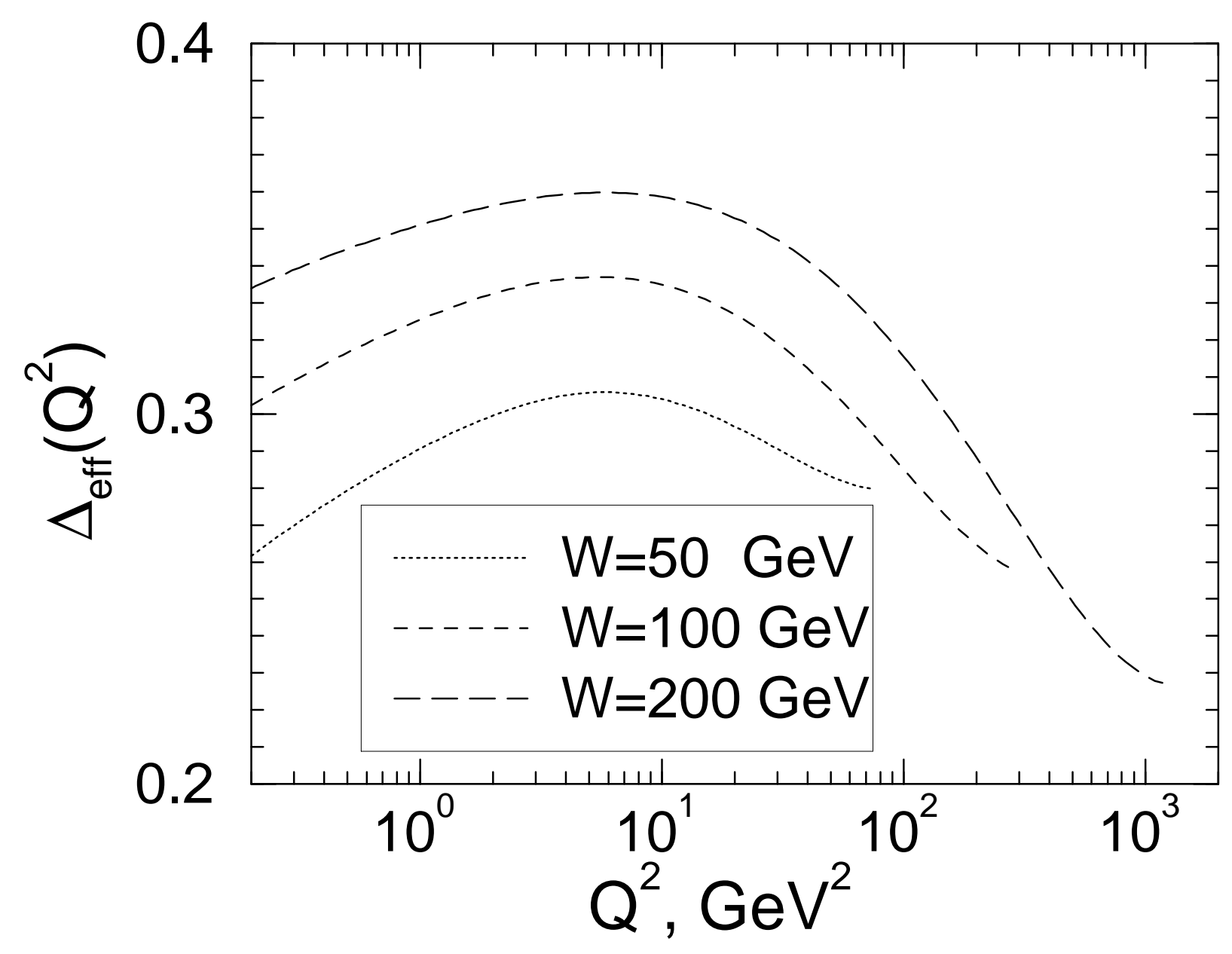




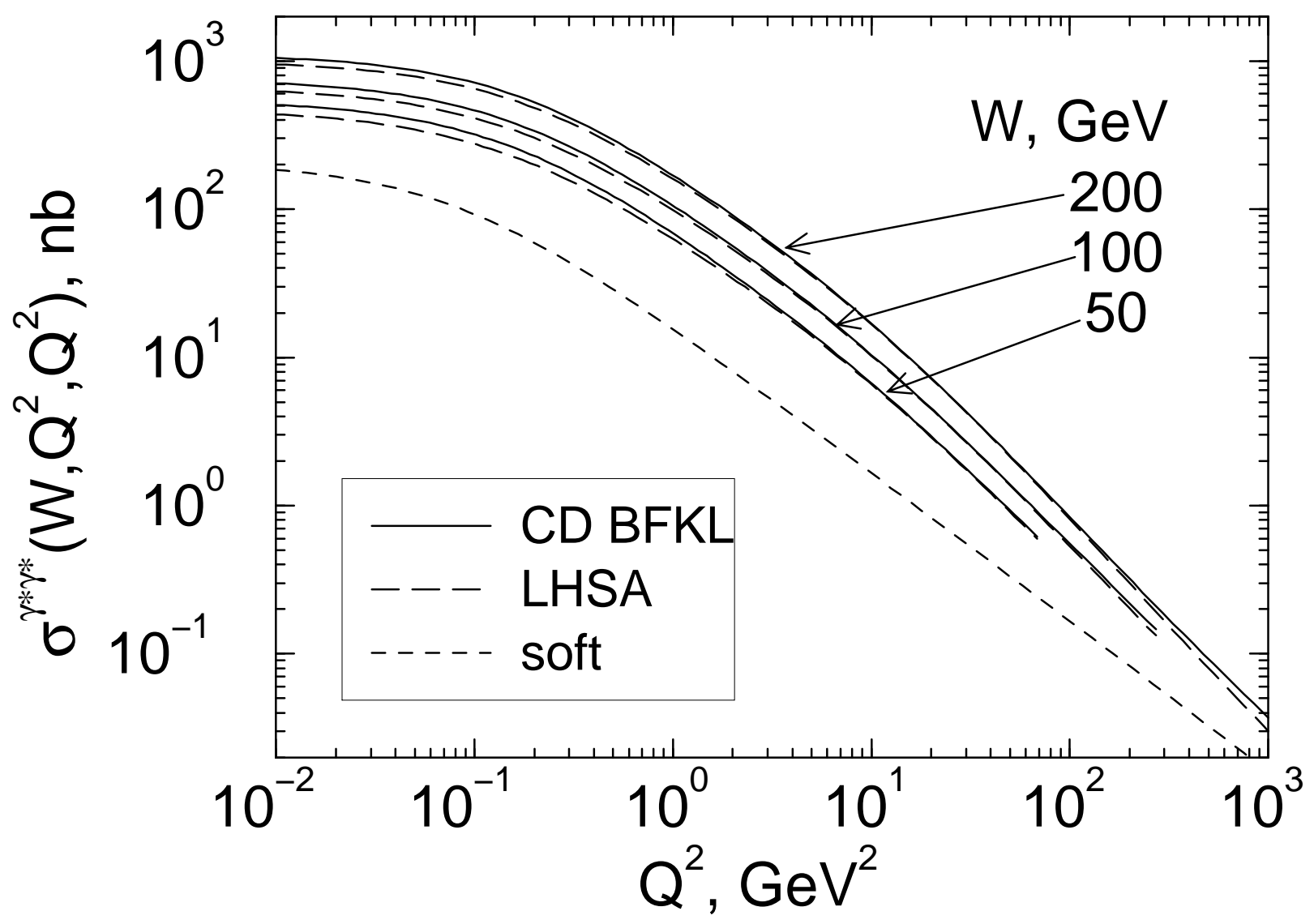



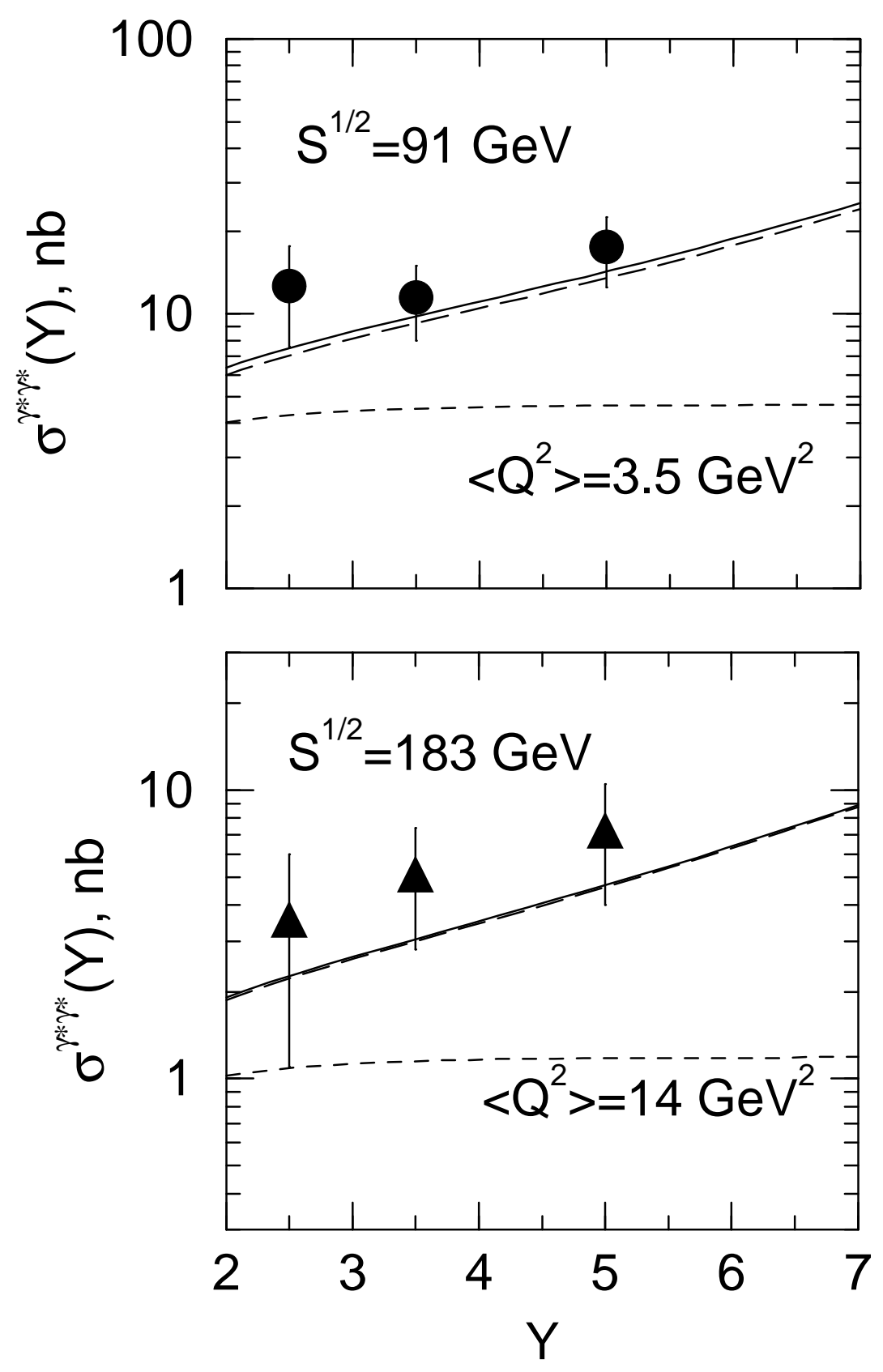


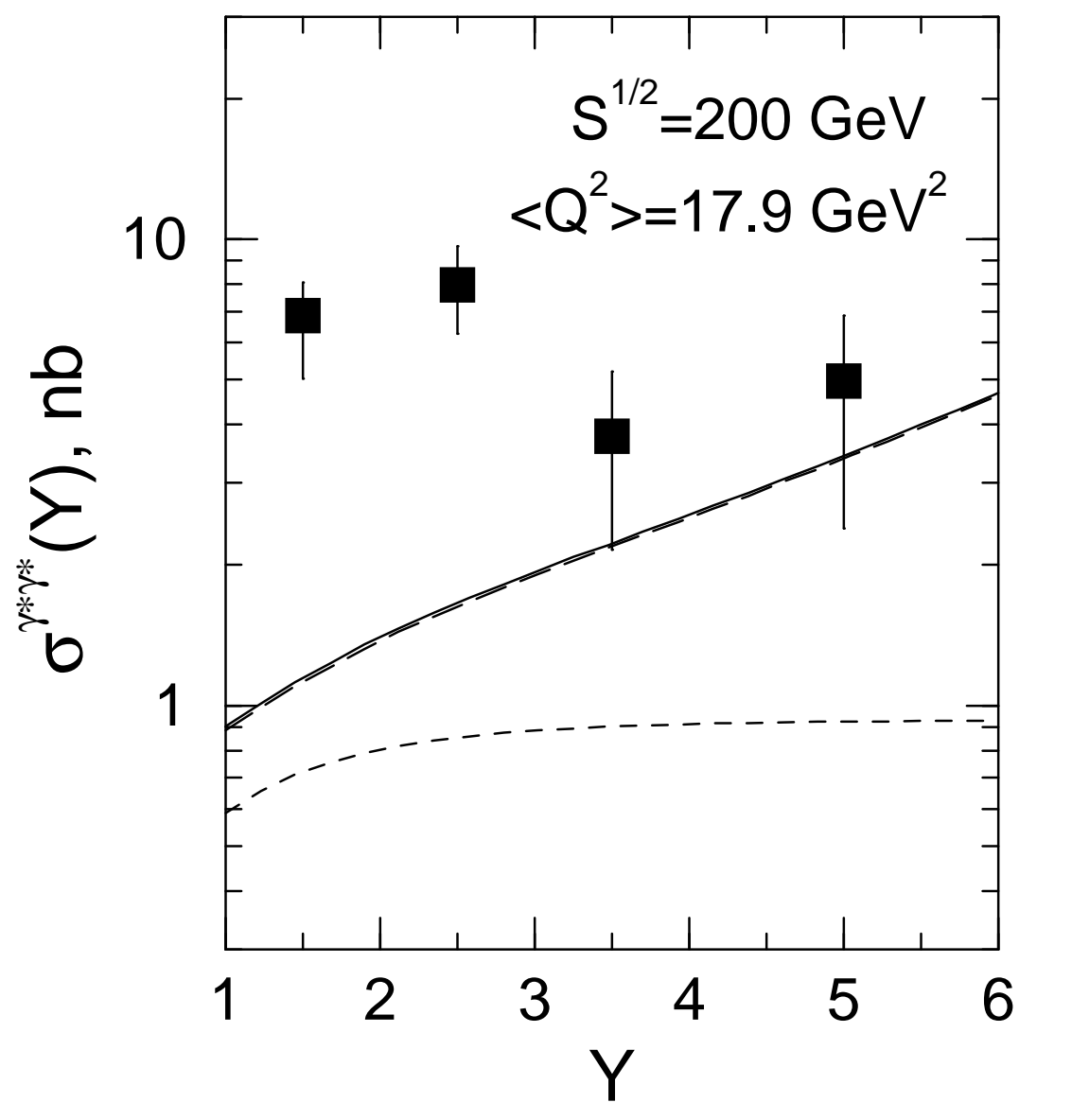



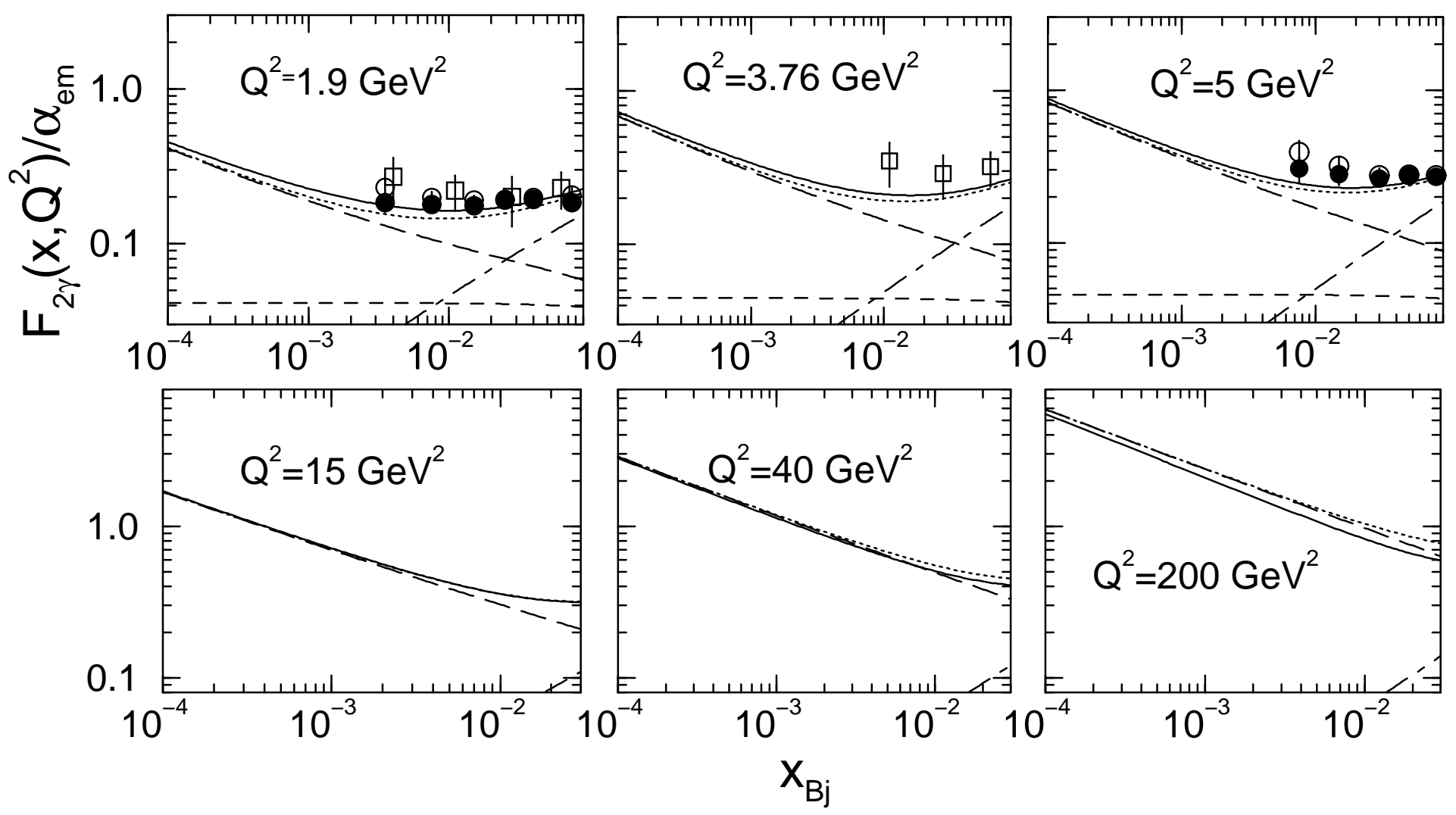


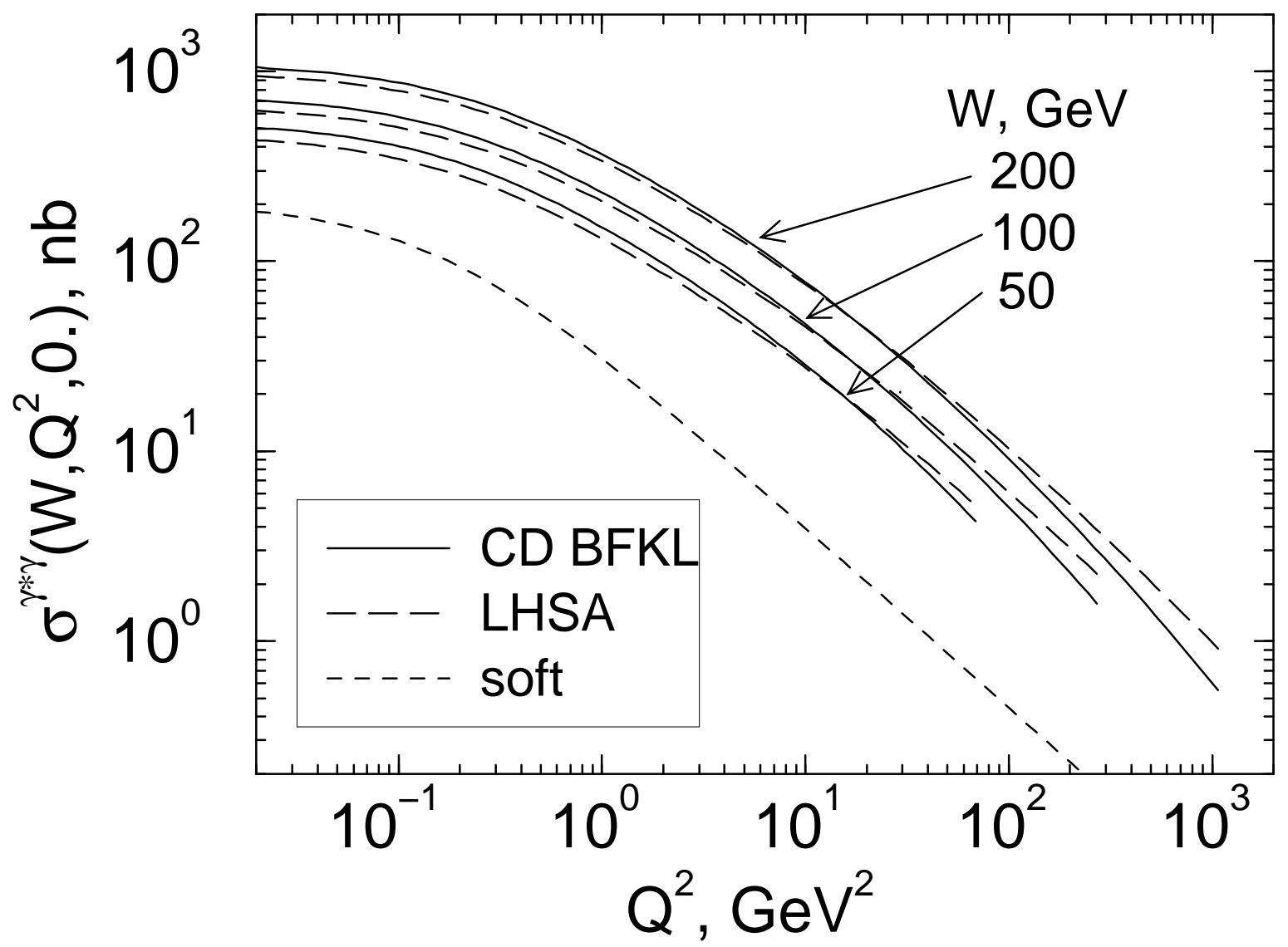




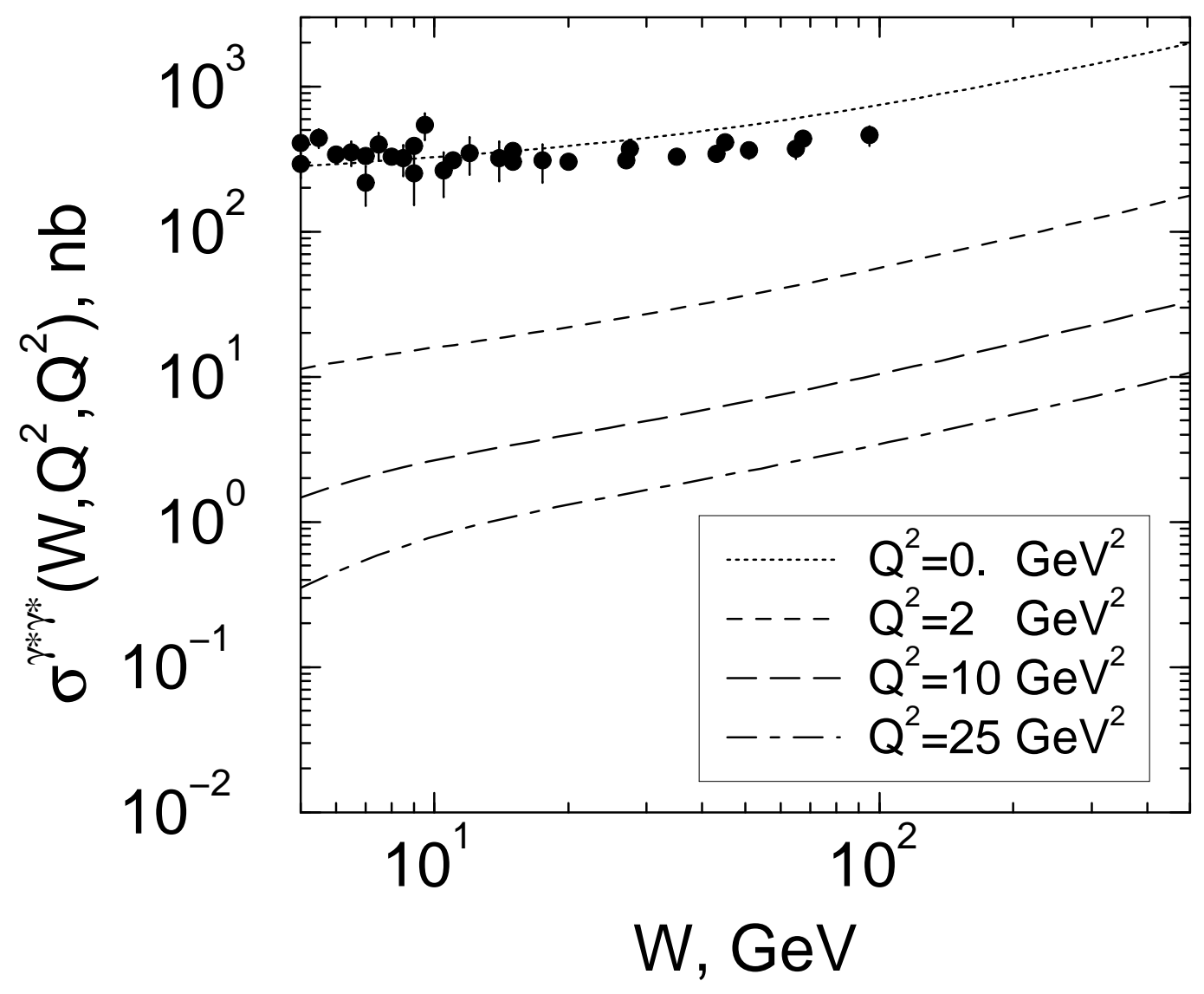




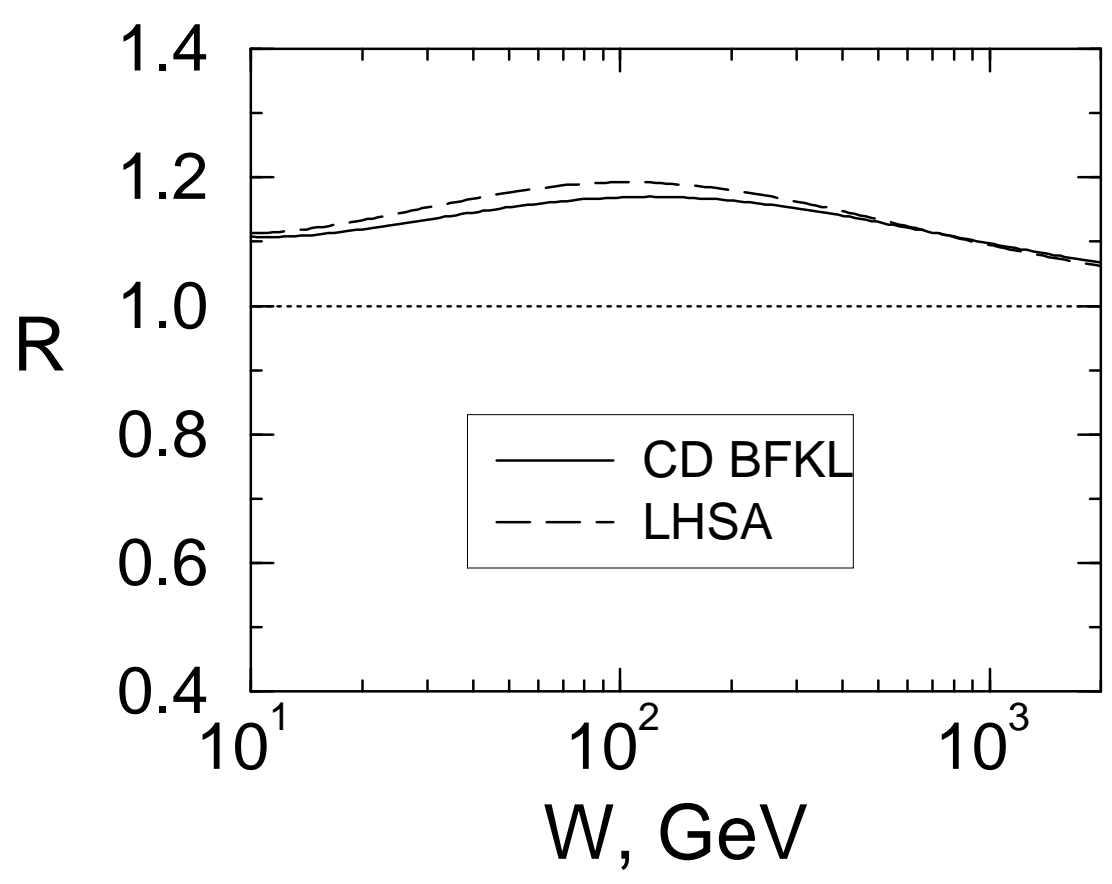




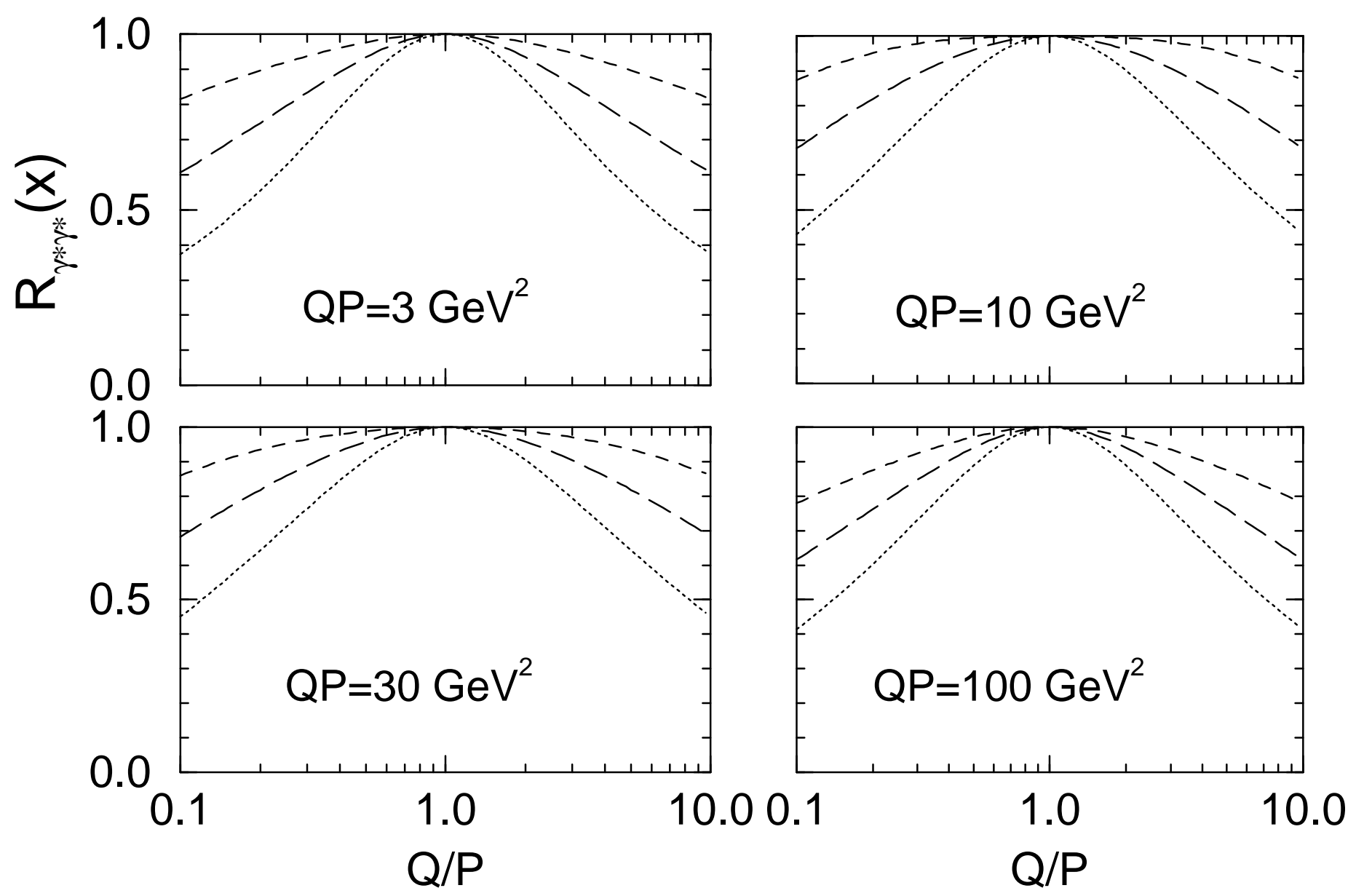

